2015

\title{
Finding Distant Galactic H li Regions
}

L. D. Anderson

W.P. Armentrout

B. M. Johnstone

T. M. Bania

Dana S. Balser

See next page for additional authors

Follow this and additional works at: https://researchrepository.wvu.edu/faculty_publications

\section{Digital Commons Citation}

Anderson, L. D.; Armentrout, W. P.; Johnstone, B. M.; Bania, T. M.; Balser, Dana S.; Wenger, Trey V.; and Cunningham, V., "Finding Distant Galactic H Ii Regions" (2015). Faculty Scholarship. 535.

https://researchrepository.wvu.edu/faculty_publications/535 
Authors

L. D. Anderson, W. P. Armentrout, B. M. Johnstone, T. M. Bania, Dana S. Balser, Trey V. Wenger, and V. Cunningham 


\title{
FINDING DISTANT GALACTIC H II REGIONS
}

\author{
L. D. Anderson ${ }^{1,2}$, W. P. Armentrout ${ }^{1}$, B. M. Johnstone ${ }^{1}$, T. M. Bania ${ }^{3}$, \\ Dana S. Balser ${ }^{4}$, Trey V. Wenger ${ }^{5}$, and V. Cunningham ${ }^{1}$ \\ ${ }^{1}$ Department of Physics and Astronomy, West Virginia University, Morgantown, WV 26506, USA \\ 2 Adjunct Astronomer at the National Radio Astronomy Observatory, P.O. Box 2, Green Bank, WV 24944, USA \\ ${ }^{3}$ Institute for Astrophysical Research, Department of Astronomy, Boston University, 725 Commonwealth Avenue, Boston, MA 02215, USA \\ ${ }_{5}^{4}$ National Radio Astronomy Observatory, 520 Edgemont Road, Charlottesville, VA 22903-2475, USA \\ ${ }^{5}$ Astronomy Department, University of Virginia, P.O. Box 3818, Charlottesville, VA 22903-0818, USA \\ Received 2015 September 11; accepted 2015 October 23; published 2015 December 1
}

\begin{abstract}
The WISE Catalog of Galactic H II Regions contains 2000 H II region candidates lacking ionized gas spectroscopic observations. All candidates have the characteristic $\mathrm{H}$ II region mid-infrared morphology of WISE $12 \mu \mathrm{m}$ emission surrounding $22 \mu \mathrm{m}$ emission, and additionally have detected radio continuum emission. We here report Green Bank Telescope hydrogen radio recombination line and radio continuum detections in the X-band $(9 \mathrm{GHz} ; 3 \mathrm{~cm})$ of 302 WISE $\mathrm{H}$ II region candidates (out of 324 targets observed) in the zone $225^{\circ} \geqslant \ell \geqslant-20^{\circ},|b| \leqslant 6^{\circ}$. Here we extend the sky coverage of our H II region Discovery Survey, which now contains nearly $800 \mathrm{H}$ II regions distributed across the entire northern sky. We provide LSR velocities for the 302 detections and kinematic distances for 131 of these. Of the 302 new detections, 5 have $(\ell, b, v)$ coordinates consistent with the Outer Scutum-Centaurus Arm (OSC), the most distant molecular spiral arm of the Milky Way. Due to the Galactic warp, these nebulae are found at Galactic latitudes $>1^{\circ}$ in the first Galactic quadrant, and therefore were missed in previous surveys of the Galactic plane. One additional region has a longitude and velocity consistent with the OSC but lies at a negative Galactic latitude (G039.183-01.422; -54.9 $\mathrm{km} \mathrm{s}^{-1}$ ). With Heliocentric distances $>22 \mathrm{kpc}$ and Galactocentric distances $>16 \mathrm{kpc}$, the OSC $\mathrm{H}$ II regions are the most distant known in the Galaxy. We detect an additional three $\mathrm{H}$ II regions near $\ell \simeq 150^{\circ}$ whose LSR velocities place them at Galactocentric radii $>19 \mathrm{kpc}$. If their distances are correct, these nebulae may represent the limit to Galactic massive star formation.
\end{abstract}

Key words: Galaxy: structure - H II regions - radio lines: ISM - surveys

Supporting material: machine-readable tables

\section{INTRODUCTION}

Galactic $\mathrm{H}$ II regions are the formation sites of massive $\mathrm{OB}$ stars. Due to their short lifetimes, $\mathrm{H}_{\text {II }}$ regions locate star formation in the present epoch and are therefore good tracers of Galactic spiral structure. H II regions are extremely bright at infrared and radio wavelengths and can be seen across the entire Galactic disk. It is perhaps surprising that the census of Galactic $\mathrm{H}$ II regions remains vastly incomplete. We have found that by combining $\mathrm{H}$ II region candidates identified at infrared (IR) wavelengths with radio spectroscopic observations, we can uncover large populations of heretofore unknown $\mathrm{H}$ II regions and determine their locations in the Galaxy.

The Green Bank Telescope H II Region Discovery Survey (GBT HRDS; Bania et al. 2010) detected radio recombination line (RRL) emission from 448 previously unknown $\mathrm{H}$ II regions in the X-band $(9 \mathrm{GHz} ; 3 \mathrm{~cm}$; Anderson et al. 2011, hereafter A11). The GBT HRDS extends over 168 square degrees, from $67^{\circ} \geqslant \ell \geqslant-17^{\circ}$ with $|b| \leqslant 1^{\circ}$. Since the ISM is optically thin at $\mathrm{cm}$-wavelengths, RRL emission can be detected from $\mathrm{H}$ II regions across the entire Galactic disk. The HRDS targets were selected based on spatially coincident $24 \mu \mathrm{m}$ (Spitzer MIPSGAL; Carey et al. 2009) and $21 \mathrm{~cm}$ continuum (VGPS; Stil et al. 2006) emission. The HRDS doubled the number of known $\mathrm{H}$ II regions in its survey zone. We followed this effort using the Arecibo Observatory and detected the RRL emission from an additional 37 sources (Bania et al. 2012) in the zone $66^{\circ} \geqslant \ell \geqslant 31^{\circ} ;|b| \leqslant 1^{\circ}$.

Because the HRDS and the Arecibo HRDS extension used the Spitzer MIPSGAL survey to identify targets, it was limited to within $1^{\circ}$ of the Galactic plane, and to within $\sim 65^{\circ}$ of the Galactic center. The HRDS had the sensitivity to discover nebulae ionized by O-stars located beyond the Solar orbit on the far side of the Milky Way (A11). Due to the Galactic warp, however, the most distant candidates are found off the Galactic plane, in regions of the Galaxy not imaged by MIPSGAL. Further, targets at the largest Galactocentric radii are generally found in the second and third Galactic quadrants. These candidates were not observed in the original HRDS.

The sensitive, high resolution mid-infrared (MIR) data required to identify new $\mathrm{H}_{\text {II }}$ regions outside of the MIPSGAL range did not exist until recently. This changed with the release of data from the Wide-field Infrared Survey Explorer (WISE). WISE has photometric bands centered at 12 and $22 \mu \mathrm{m}$, providing spectral coverage analogous to the 8.0 and $24 \mu \mathrm{m}$ Spitzer bands. Using these WISE data, Anderson et al. (2014, hereafter A14) created a catalog of over $8000 \mathrm{H}$ II regions and candidates spanning all Galactic longitudes within $8^{\circ}$ of the Galactic midplane. All these objects have the MIR emission identified in A11 as being characteristic of $\mathrm{H}_{\mathrm{II}}$ regions. About 2000 cataloged objects also have spatially coincident radio continuum emission, and as A11 found, this makes them strong candidates for being bona fide $\mathrm{H}$ II regions. Because of its sensitivity and all-sky coverage, WISE can detect the MIR emission from all Galactic $\mathrm{H}_{\text {II }}$ regions ionized by single O-stars (A14).

By extending the latitude coverage of the $\mathrm{H}$ it region surveys, we can detect extremely distant massive star formation regions, in particular in the Outer Scutum-Centaurus Arm (OSC). The 
OSC is the most distant molecular spiral arm known, but it is little studied because it is located primarily at Galactic latitudes $>1^{\circ}$ in the first Galactic quadrant (Dame \& Thaddeus 2011). Aside from S83 (G55.11+2.4), no massive star formation was known in the OSC before the HRDS. The OSC arm appears to be the Outer Galaxy continuation of the Scutum-Centaurus Arm, and is $\sim 15 \mathrm{kpc}$ from the Galactic center at a Heliocentric distance of $\sim 20 \mathrm{kpc}$.

Here we provide a catalog of RRL and radio continuum properties for $302 \mathrm{H}$ II regions detected out of 324 targets identified in the WISE Catalog of Galactic H II regions.

\section{OBSERVATIONS AND DATA ANALYSIS}

We draw our targets from the MIR objects in the WISE catalog of A14. Most targets are identified in the WISE catalog as "candidates" that have detected radio continuum emission, but we also include in our sample Sharpless $\mathrm{H}$ II regions (Sharpless 1959) that have never been observed in RRL emission. All Sharpless regions observed here were observed in $\mathrm{H} \alpha$ by Fich et al. (1990), but at a spectral resolution of just $15 \mathrm{~km} \mathrm{~s}^{-1}$. This low spectral resolution may lead to inaccurate line parameters (see Section 4.1). We used the entire zone of the sky visible by the GBT, north of $-42^{\circ}$ decl. This corresponds to approximately $270^{\circ}>\ell>-20^{\circ}$ at $b=0^{\circ}$, although we did not detect any $\mathrm{H}_{\text {II }}$ regions within $270^{\circ}>\ell>225^{\circ}$. All targets have peak radio continuum emission of at least $30 \mathrm{mJy}$ (extrapolated to X-band [3 cm] wavelengths assuming optically thin free-free continuum emission, $S_{\nu} \propto \nu^{-0.1}$ ). This flux limit is considerably less than that of the original HRDS, $70 \mathrm{mJy}$. This results in a number of target candidates within the original HRDS longitude and latitude zone. To get the extrapolated X-band flux densities, we measure the peak continuum flux densities using the VLA Galactic Plane Survey continuum data at $21 \mathrm{~cm}$ (VGPS; Stil et al. 2006), the Canadian Galactic Plane Survey continuum data at $21 \mathrm{~cm}$ (CGPS; Taylor et al. 2003), the NRAO VLA Sky Survey at $20 \mathrm{~cm}$ (NVSS; Condon et al. 1998), the SuperMongolo Sky Survey at $36 \mathrm{~cm}$ (SUMSS; Bock et al. 1999), and the Southern Galactic Plane Survey (SGPS; McClure-Griffiths et al. 2005) at $21 \mathrm{~cm}$, depending on the candidate $(\ell, b)$ position.

For $65^{\circ} \geqslant \ell \geqslant 25^{\circ}, b \geqslant 1^{\circ}$, we include in our target list all $\mathrm{H}$ II region candidates with detected radio continuum emission, regardless of their flux densities. This is the $(\ell, b)$ zone of the OSC (Dame \& Thaddeus 2011; see above). Sources in this Galactic region have a higher likelihood of being extremely distant and therefore fainter than the rest of the sample.

We followed the same GBT observational procedure as in the original HRDS: interleaving total power spectral line (using the AutoCorrelation Spectrometer (ACS)) and total power continuum (using the Digital Continuum Receiver (DCR)) observations for each candidate $\mathrm{H}_{\text {II }}$ region. Our observations were made with the GBT $100 \mathrm{~m}$ telescope from 2012 July through 2014 August. There are seven RRLs that can be cleanly observed simultaneously with the GBT in the X-band: $\mathrm{H} 87 \alpha$ to $\mathrm{H} 93 \alpha$. We average these seven RRLs (each at two orthogonal polarizations) to create a single average RRL spectrum.

For the RRL observations, we call each set of total power observations a "pair." These pairs consist of 6 minute on- and 6 minute off-source integrations, and the off-source position tracks the same path on the sky as the on-source position. In the original HRDS, a single position-switched pair had an average rms noise of $\sim 1 \mathrm{mJy}$ after smoothing to $1.86 \mathrm{~km} \mathrm{~s}^{-1}$ (see below), and the rms noise was $\sim 0.7 \mathrm{mJy}$ for two pairs. Assuming a line-to-continuum intensity ratio of 0.1 (Quireza et al. 2006a), our $30 \mathrm{mJy}$ cutoff corresponds to a $3 \sigma$ detection in a single pair. We observe a single pair for all sources and do additional pairs as needed.

As in the HRDS, the continuum observations consisted of four cross scans centered on the nominal source position: forward and backward in right ascension (R.A.) and forward and backward in declination (decl.). Each scan was $40^{\prime}$ in length and had a slew rate of $80^{\prime \prime}$ per second. We observed at a center frequency of $8665 \mathrm{MHz}$ with a $320 \mathrm{MHz}$ bandwidth. We focused the telescope and established local pointing corrections on average every two hours using standard X-band pointing sources.

In A11, observations of flux calibrators shows that the intensity scale was accurate at the $10 \%$ level for RRL and continuum data. We established the calibration of the intensity scale using noise diodes fired during data acquisition. We repeat the same calibration procedure for these data, using $80 \mathrm{MHz}$ continuum cross-scans in the R.A. and decl. directions of the calibrator $3 \mathrm{C} 147$ using the DCR at frequencies from 8.04 to $9.81 \mathrm{GHz}$. At $8.3 \mathrm{GHz}$ the peak intensity of $3 \mathrm{C} 147$ averaged over both polarizations and all cross scans is $9.81 \mathrm{~K}$, or $4.90 \mathrm{Jy}$ using the GBT X-band gain of $2 \mathrm{~K} \mathrm{Jy}^{-1}$. This value is within $5 \%$ of the peak flux density at $8.3 \mathrm{GHz}$ for $3 \mathrm{C} 147$ given in Peng et al. (2000). The flux densities at the other continuum frequencies agree to within $10 \%$ of that expected for $3 \mathrm{C} 147$ assuming a spectral index, $\alpha$, of $0.91 \pm 0.03$, where $S_{\nu} \propto \nu^{-\alpha}$ (Peng et al. 2000). The X-band intensity scale is relatively insensitive to opacity and elevation gain corrections, which both have magnitudes of $\lesssim 5 \%$ (Ghigo et al. 2001). Because we are making a discovery survey, we did not correct our data based on the intensity of 3C147 or for gain and opacity variations. We therefore estimate, as in the original HRDS, that the intensities and flux densities given in this paper are uncertain at the $10 \%$ level.

We follow the same data reduction steps as in the original HRDS. We again use the TMBIDL software package (Bania et al. 2014, V7.1, see also https://github.com/tvwenger/ tmbidl). For the RRL data, we average the seven RRL transitions with two polarizations to create a single $\langle\mathrm{Hn} \alpha\rangle$ spectrum (see Balser 2006). Averaging the spectra in this way improves the RRL signal-to-noise ratio, allowing for significantly reduced integration times. We smooth each combined $\langle\mathrm{Hn} \alpha\rangle$ spectrum with a normalized Gaussian over five channels to give a $\langle\mathrm{H} \mathrm{n} \alpha\rangle$ spectrum with a velocity resolution of $1.86 \mathrm{~km} \mathrm{~s}^{-1}$. The original HRDS found hydrogen line widths of $\sim 25 \mathrm{~km} \mathrm{~s}^{-1}$ on average, giving us $\gtrsim 10$ independent spectral channels per line after this smoothing. We remove a polynomial baseline, typically of third-order, and fit Gaussians to each detected hydrogen line component individually for each source. As in the HRDS, we assume that the brightest line is from hydrogen. ${ }^{6}$ Thus, we derive the $\mathrm{LSR}^{7}$ velocity, line intensity, and FWHM line width for each hydrogen RRL

\footnotetext{
6 There are, however, a few sources that have carbon RRLs brighter than those of hydrogen. These are easily distinguished because the carbon lines are $\lesssim 10 \mathrm{~km} \mathrm{~s}^{-1}$ wide (Wenger et al. 2013), and are offset $\sim-150 \mathrm{~km} \mathrm{~s}^{-1}$ from the hydrogen lines.

7 We used the kinematic local standard of rest (LSR) frame with the radio definition of the Doppler shift. The kinematic LSR is defined by a solar motion of $20.0 \mathrm{~km} \mathrm{~s}^{-1}$ toward $(\alpha, \delta)=\left(18^{\mathrm{h}},+30^{\circ}\right)$ [1900.0] (Gordon 1976).
} 

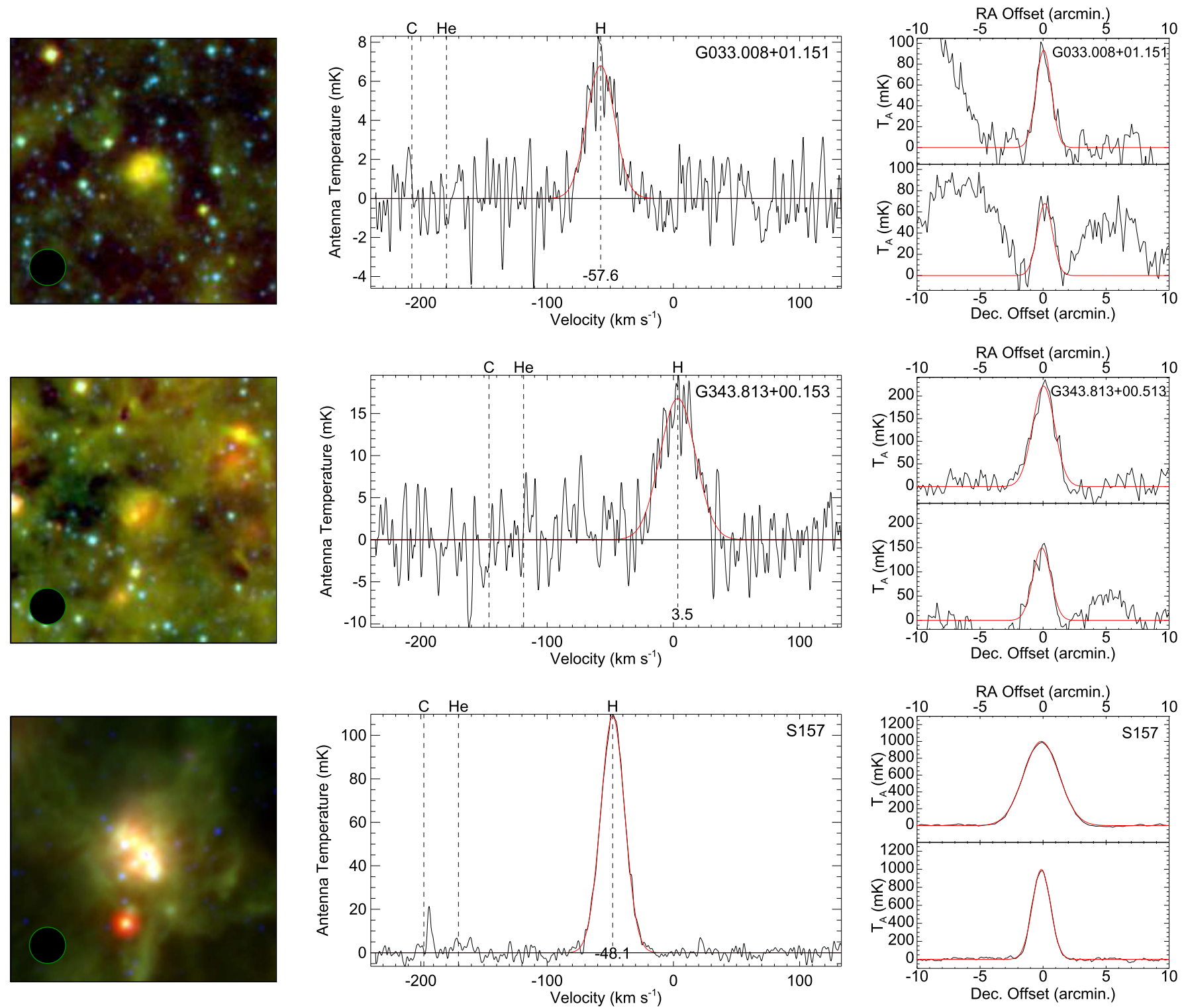

Figure 1. Example H II regions G033.006+01.151 (top row), G343.813+00.153 (middle), and S157 (bottom). The left column shows WISE $22 \mu \mathrm{m}$ emission in red, WISE $12 \mu \mathrm{m}$ emission in green, and WISE $3.6 \mu \mathrm{m}$ emission in blue. Each image is $10^{\prime}$ on a side oriented in Galactic coordinates and the 82" GBT beam is shown in the lower left. $\mathrm{H}$ II region candidates were identified based on their characteristic MIR morphology. The middle column shows average X-band $\langle\mathrm{H} n \alpha\rangle$ spectra. The spectra have been smoothed to $1.86 \mathrm{~km} \mathrm{~s}^{-1}$ resolution and the hydrogen Gaussian fits are shown in red. The expected velocities of the helium and carbon RRLs are also indicated. The right column shows continuum cross scans in R.A. (top panel) and decl. (bottom panel). Gaussian fits to the sources are shown in red.

component. We did not correct for the changing beam size with frequency and therefore the line parameters are averages over beam sizes from $73^{\prime \prime}$ to $89^{\prime \prime}$.

We average the forward and backward continuum scans for each source (after first flipping the backwards scans) to create a R.A. scan and a decl. scan. We remove a polynomial baseline, usually a second-order, from the two scans and then fit Gaussians, again on a source-by-source basis. Some sources have multiple emission components, which we fit with multiple Gaussians. Using WISE and radio continuum data as a guide, we attempt to associate a single Gaussian component with the source observed (see A11 for a more complete discussion). As A11 note, source confusion is a significant issue and the continuum parameters should be used with caution.

We show in Figure 1 for three example $\mathrm{H}$ II regions the WISE three color images $(22 \mu \mathrm{m}$ in red, $12 \mu \mathrm{m}$ in green, and $3.6 \mu \mathrm{m}$ in blue), $\langle\mathrm{Hn} \alpha\rangle$ spectra, and continuum cross scans. These three regions are representative of the sample as a whole in terms of angular size, spectral line intensity, and continuum data quality.

\section{THE CATALOG OF WISE-IDENTIFIED H II REGIONS}

We detect RRL emission from 302 of the 324 targets, over $93 \%$ of our sample. Excluding the 31 regions in the $(\ell, b)$ zone of the $\operatorname{OSC}\left(65^{\circ}>\ell>25^{\circ} ; b \geqslant 1^{\circ}\right)$ where our flux density threshold was relaxed, these numbers change to 280 of 293 (96\%). In both cases, these values are similar to the $95 \%$ detection rate of A11. The hydrogen RRL data are given in Table 1, which lists the source name, the Galactic longitude and latitude, the line intensity, the FWHM line width, the LSR velocity, and the rms noise in the spectrum. The errors given in Table 1 for the line parameters are the $1 \sigma$ uncertainties from the Gaussian fits. For sources with multiple velocity 
Table 1

Hydrogen Recombination Line Parameters

\begin{tabular}{|c|c|c|c|c|c|c|c|c|c|c|}
\hline Source & $\begin{array}{l}\ell \\
\left({ }^{\circ}\right)\end{array}$ & $\begin{array}{l}b \\
\left({ }^{\circ}\right)\end{array}$ & $\begin{array}{c}T_{L} \\
(\mathrm{mK})\end{array}$ & $\begin{array}{c}\sigma T_{L} \\
(\mathrm{mK})\end{array}$ & $\begin{array}{c}\Delta V \\
\left(\mathrm{~km} \mathrm{~s}^{-1}\right)\end{array}$ & $\begin{array}{c}\sigma \Delta V \\
\left(\mathrm{~km} \mathrm{~s}^{-1}\right)\end{array}$ & $\begin{array}{c}V_{\mathrm{LSR}} \\
\left(\mathrm{km} \mathrm{s}^{-1}\right)\end{array}$ & $\begin{array}{c}\sigma V_{\mathrm{LSR}} \\
\left(\mathrm{km} \mathrm{s}^{-1}\right)\end{array}$ & $\begin{array}{l}\mathrm{rms} \\
(\mathrm{mK})\end{array}$ & Note $^{\mathrm{a}}$ \\
\hline G000.335-00.015a & 0.335 & -0.015 & 164.9 & 1.7 & 33.7 & 0.5 & 14.0 & 0.3 & 3.1 & * \\
\hline G000.335-00.015b & 0.335 & -0.015 & 47.5 & 1.8 & 32.2 & 1.8 & 46.5 & 1.1 & 3.1 & \\
\hline G000.492+00.192 & 0.492 & +0.192 & 106.0 & 0.5 & 22.1 & 0.1 & -7.5 & 0.1 & 2.6 & \\
\hline G000.583-00.870 & 0.583 & -0.870 & 281.4 & 2.2 & 17.4 & 0.2 & 14.2 & 0.1 & 2.4 & \\
\hline G000.602-00.051 & 0.602 & -0.051 & 568.2 & 2.5 & 41.1 & 0.2 & 53.1 & 0.1 & 2.3 & \\
\hline G000.630-00.027a & 0.630 & -0.027 & 188.2 & 0.3 & 52.8 & 0.5 & 90.6 & 0.1 & 3.3 & \\
\hline G000.630-00.027b & 0.630 & -0.027 & 87.2 & 0.7 & 37.0 & 0.4 & 51.3 & 0.2 & 3.3 & \\
\hline G000.630-00.027c & 0.630 & -0.027 & 27.9 & 0.5 & 28.8 & 0.6 & 136.2 & 0.3 & 3.3 & \\
\hline G000.639-00.114a & 0.639 & -0.114 & 192.6 & 1.1 & 27.4 & 0.4 & 70.4 & 0.1 & 3.3 & \\
\hline G000.639-00.114b & 0.639 & -0.114 & 65.3 & 1.1 & 27.6 & 0.6 & 24.9 & 0.2 & 3.3 & \\
\hline G000.639-00.114c & 0.639 & -0.114 & 33.4 & 1.7 & 17.4 & 1.2 & 96.7 & 0.6 & 3.3 & \\
\hline G000.670-00.034 & 0.670 & -0.034 & 2047.0 & 5.9 & 40.2 & 0.1 & 64.8 & 0.1 & 11.7 & \\
\hline G000.682-00.035 & 0.682 & -0.035 & 1804.1 & 5.0 & 39.1 & 0.1 & 66.4 & 0.1 & 5.7 & \\
\hline G000.692-00.046 & 0.692 & -0.046 & 504.5 & 1.2 & 40.9 & 0.1 & 60.0 & 0.1 & 3.9 & \\
\hline G001.005-00.236 & 1.005 & -0.236 & 32.2 & 0.4 & 22.4 & 0.3 & 2.5 & 0.1 & 2.4 & \\
\hline
\end{tabular}

Note.

${ }^{a}$ Discrete $\mathrm{H}$ II region velocity for sources with multiple velocity component spectra marked with an asterisk $\left(^{*}\right)$.

(This table is available in its entirety in machine-readable form.)

Table 2

Radio Continuum Parameters

\begin{tabular}{|c|c|c|c|c|c|c|c|c|c|c|c|c|c|}
\hline Source & $\begin{array}{l}\ell \\
\left(^{\circ}\right)\end{array}$ & $\begin{array}{l}b \\
\left({ }^{\circ}\right)\end{array}$ & $\begin{array}{r}T_{\alpha} \\
(\mathrm{mK})\end{array}$ & $\begin{array}{r}\sigma T_{\alpha} \\
(\mathrm{mK})\end{array}$ & $\begin{array}{r}T_{\delta} \\
(\mathrm{mK})\end{array}$ & $\begin{array}{r}\sigma T_{\delta} \\
(\mathrm{mK})\end{array}$ & $\begin{array}{l}\Theta_{\alpha} \\
\left({ }^{\prime \prime}\right)\end{array}$ & $\begin{array}{r}\sigma \Theta_{\alpha} \\
\left({ }^{\prime \prime}\right)\end{array}$ & $\begin{array}{l}\Theta_{\delta} \\
\left({ }^{\prime \prime}\right)\end{array}$ & $\begin{array}{c}\sigma \Theta_{\delta} \\
\left({ }^{\prime \prime}\right)\end{array}$ & $\begin{array}{r}S \\
(\mathrm{mJy})\end{array}$ & $\begin{array}{r}\sigma S \\
(\mathrm{mJy})\end{array}$ & Note $^{\mathrm{a}}$ \\
\hline G000.335-00.015 & 0.335 & -0.015 & 2101 & 17 & 2109 & 29 & 129 & 2 & 111 & 3 & 2002 & 157 & $\mathrm{P}$ \\
\hline G000.492+00.192 & 0.492 & +0.192 & 673 & 13 & 628 & 7 & 134 & 5 & 105 & 2 & 604 & 59 & $\cdots$ \\
\hline G000.602-00.051 & 0.602 & -0.051 & 7709 & 86 & 7947 & 55 & 173 & 3 & 135 & 2 & 12091 & 835 & $\mathrm{P}$ \\
\hline G000.630-00.027 & 0.630 & -0.027 & $\ldots$ & $\ldots$ & 4951 & 21 & $\ldots$ & $\ldots$ & 90 & 1 & 5147 & 74 & $\ldots$ \\
\hline G000.639-00.114 & 0.639 & -0.114 & $\ldots$ & $\cdots$ & 2116 & 49 & $\cdots$ & $\cdots$ & 116 & 2 & 2832 & 111 & $\cdots$ \\
\hline G000.692-00.046 & 0.692 & -0.046 & $\ldots$ & $\cdots$ & 6560 & 62 & $\cdots$ & $\cdots$ & 115 & 3 & 8646 & 237 & $\cdots$ \\
\hline G001.005-00.236 & 1.005 & -0.236 & 481 & 8 & 458 & 12 & 178 & 6 & 147 & 8 & 809 & 144 & ... \\
\hline
\end{tabular}

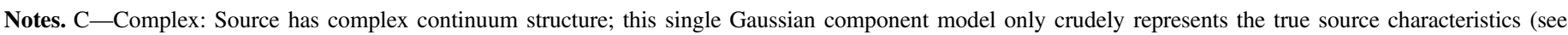
text). P-Peaked: Continuum peak lies within $10^{\prime \prime}$ of the nominal target position. These are the highest quality continuum data.

a Comments concerning continuum emission morphology and data quality.

(This table is available in its entirety in machine-readable form.)

components detected along the line of sight, we append to their names additional letters "a," "b," or "c" in order of decreasing peak line intensity. For multiple-velocity sources, if we were able to determine which component stems from the discrete $\mathrm{H}$ II region (see Section 3.1) it is flagged with an asterisk in the final column of Table 1.

We give the radio continuum data in Table 2 , which lists the source name, the Galactic longitude and latitude, the peak intensity in the R.A. and decl. scan directions, the FWHM angular size in the R.A. and decl. directions, the integrated flux density, and the notes on continuum morphology (see below). We compute the integrated flux density as in A11:

$$
S_{i}=S_{p}\left(\frac{\theta_{\alpha}}{\theta_{b}}\right)\left(\frac{\theta_{\delta}}{\theta_{b}}\right),
$$

where $S_{p}$ is the peak flux found from the peak antenna temperature assuming a gain of $2 \mathrm{~K} \mathrm{Jy}^{-1}, \theta_{b}$ is the GBT beam size at $8665 \mathrm{MHz}\left(87^{\prime \prime}\right)$, and $\theta_{\alpha}, \theta_{\delta}$ are the FWHM angular sizes derived from the R.A. and decl. scans (see Kuchar \& Clark 1997). As in A11, for sources better approximated by multiple Gaussians, the angular size listed is the maximum extent of the multi-component composite source and the peak intensity (e.g., $T_{\alpha}$ ) is the area under all components divided by this angular size. Such "complex" objects are marked with a "C" label in the Notes column of Table 2. The errors given for the continuum parameters are $1 \sigma$ uncertainties in the Gaussian fits. We flag nebulae whose peak continuum emission is within 10 " of the nominal target position with a "P" label in the Notes column of Table 2. These are our highest quality continuum data, and for these sources alone the continuum data may be suitable for deriving physical properties. Due to confusion or poor data quality, some sources were not detected in either or both continuum cross-scans and in such cases we do not provide continuum intensities or angular sizes. 


\subsection{Multiple-velocity H II Regions}

Of the 302 detections, 57 have spectra with multiple hydrogen RRL components at different velocities. In total, we detect the emission from 369 RRL components (245 with one line, 47 sources with two lines, and 10 sources with three lines). As in Anderson et al. (2015, hereafter A15) we hypothesize that one of these component is from the discrete $\mathrm{H}$ II region that we targeted and the other(s) are from diffuse ionized gas along the line of sight. To compute kinematic distances, we must determine the correct RRL velocity for these multiple-velocity $\mathrm{H}$ II region spectra.

In A15 we derived a set of criteria that can be used to determine which of the RRL components is from the discrete $\mathrm{H}$ II region. From the data given here, we can use four of their criteria: (1) the presence of a negative RRL velocity component, the association between RRL velocities and those of either (2) molecular gas or (3) carbon RRLs, and (4) an analysis of the electron temperature for each RRL velocity component. As in A15, we require that the results from all four criteria agree with each other. Below we discuss the four criteria, and how we apply them to our data set.

For the first of the four criteria, A15 argue that diffuse ionized gas is unlikely to be found in the outer Galaxy, at negative velocities in the first Galactic quadrant, since the density of free electrons in the outer Galaxy is low (e.g., Taylor \& Cordes 1993). As in A15 we therefore assume that for all first-quadrant multiple-velocity $\mathrm{H}_{\text {II }}$ regions the negative velocity components are from the discrete $\mathrm{H}$ II regions.

Two of the four criteria rely on the detection of other spectral lines within $10 \mathrm{~km} \mathrm{~s}^{-1}$ of one of the $\langle\mathrm{H} \mathrm{n} \alpha\rangle$ velocity components. A15 argue that molecular gas and carbon RRLs are more likely to be associated with discrete $\mathrm{H}$ II regions than diffuse ionized gas. For each multiple-velocity $\mathrm{H}$ II region, we correlate the molecular velocities compiled by A14 with the $\langle\mathrm{Hn} \alpha\rangle$ velocities measured here. We search for carbon lines by examining each spectrum for emission offset $\sim-150 \mathrm{~km} \mathrm{~s}^{-1}$ from the hydrogen RRL velocity that is at least three times the rms, and correlate any such sources with the $\langle\operatorname{Hn} \alpha\rangle$ velocities. These correlations produce lists of velocity components with associated molecualar gas or carbon RRLs, which we assume are the discrete $\mathrm{H}$ II region velocities.

Finally, A15 use the derived electron temperature values, $T_{e}$, for each RRL component to determine the discrete $\mathrm{H}$ II region RRL components. In local thermodynamic equilibrium (LTE), the electron temperature can be computed from observable quantities:

$$
\begin{aligned}
T_{e}= & 7103.3\left(\frac{\nu}{\mathrm{GHz}}\right)^{1.1}\left[\frac{T_{C}}{T_{L}\left(\mathrm{H}^{+}\right)}\right] \\
& \times\left[\frac{\Delta v\left(\mathrm{H}^{+}\right)}{\mathrm{km} \mathrm{s}^{-1}}\right]^{-1}\left[1+\frac{n\left({ }^{4} \mathrm{He}^{+}\right)}{n\left(\mathrm{H}^{+}\right)}\right]^{-1},
\end{aligned}
$$

where $\nu$ is the observing frequency, $T_{C} / T_{L}$ is the continuum-toline intensity ratio, $\Delta v$ is the RRL FWHM line width, and $n\left({ }^{4} \mathrm{He}^{+}\right) / n\left(\mathrm{H}^{+}\right)$is the helium ionic abundance ratio by number, $y^{+}$. Each multiple-velocity $\mathrm{H}$ II region has a single value for $T_{C}$, but multiple values for $T_{L}$ and $\Delta v$, and therefore has a different $T_{e}$ for each RRL component. Assuming that only one of the detected lines is from a discrete $\mathrm{H}$ II region, the line-tocontinuum intensity ratio is only physically meaningful for this
Table 3

H II Region Kinematic Distance Summary ${ }^{a}$

\begin{tabular}{lcc}
\hline \hline Category & Previously Known & This Work \\
\hline Inner Galaxy $\left(R_{G}<8.5 \mathrm{kpc}\right)$ & 661 & 26 \\
Sharpless (Near Distance) & 17 & 4 \\
Tangent Point & 166 & 6 \\
KDAR & 478 & 16 \\
Outer Galaxy $\left(R_{G}>8.5 \mathrm{kpc}\right)$ & 160 & 105 \\
1st Quadrant & 54 & 34 \\
2nd Quadrant & 72 & 66 \\
3rd Quadrant $\left(\ell<225^{\circ}\right)$ & 27 & 1 \\
4th Quadrant $\left(\ell>340^{\circ}\right)$ & 7 & 4 \\
Total & 821 & 131 \\
\hline
\end{tabular}

Note.

a Table only includes $\mathrm{H}$ II regions with known distances. The "Previously Known" values are from A14.

component. Therefore, whereas the electron temperature can be computed for all detected lines for each multiple-velocity $\mathrm{H}$ II region, the line from the $\mathrm{H}_{\text {II }}$ region should have a reasonable value of $T_{e}$ while the others may not. Based on their analysis of single-velocity $\mathrm{H}_{\mathrm{II}}$ regions from the HRDS, A15 define this "reasonable" range of $T_{e}$ values (in Kelvin) as a function of Galactocentric radius, $R_{G}(\mathrm{kpc})$, for high quality sources to be: $860+544 R_{G}<T_{e}<2040+544 R_{G}$. A high quality source is one with a peak continuum intensity of at least $100 \mathrm{mK}$, a peak within $10^{\prime \prime}$ of the nominal centroid pointing, and radio continuum emission that can be modeled with a single Gaussian component. A range for low-quality sources not meeting at least one of the above criteria is defined by A15 to be: $T_{e}<6300+544 R_{G}$. Here, we compute a value of $T_{e}$ for each detected hydrogen component using $8.7 \mathrm{GHz}$ for $\nu$, and assuming $y^{+}=0.07$ (Quireza et al. 2006b). We use the "reasonable" range of values above to identify which RRLs originate from discrete $\mathrm{H}$ II regions.

\subsection{Distances}

We derive kinematic distances for 131 of the detected $\mathrm{H}$ II regions. Of these, 105 are outer-Galaxy regions $\left(R_{G}>8.5 \mathrm{kpc}\right)$ and 26 are inner-Galaxy regions. The results of our kinematic distance analysis, in addition to the numbers of regions known previously, are summarized in Table 3.

Kinematic distances use a model for Galactic rotation to give distances as a function of observed velocity for a given line of sight. We derive all kinematic distance parameters using the Brand \& Blitz (1993) rotation curve. Kinematic distances are prone to large uncertainties in certain parts of the Galaxy. As in previous work, we estimate kinematic distance uncertainties by adding in quadrature the uncertainties associated with the rotation curve choice, streaming motions of $7 \mathrm{~km} \mathrm{~s}^{-1}$, and changes to the Solar circular rotation speed. Such uncertainties were first computed by Anderson et al. (2012a), and expanded to the entire Galaxy by A14. We use the latter analysis here.

Kinematic distances are possible for 185 nebulae. We do not compute kinematic distances for sources within $10^{\circ}$ of the Galactic center or within $20^{\circ}$ of the Galactic anti-center because such distances would be uncertain by $\gtrsim 50 \%$ (A14). We also exclude $\mathrm{H}_{\text {II }}$ regions with multiple RRL velocities for which the 
Table 4

Kinematic Distances

\begin{tabular}{|c|c|c|c|c|c|c|c|c|c|c|}
\hline Name & $\begin{array}{c}V_{\mathrm{LSR}} \\
\left(\mathrm{km} \mathrm{s}^{-1}\right)\end{array}$ & $\begin{array}{c}D_{N} \\
(\mathrm{kpc})\end{array}$ & $\begin{array}{c}D_{F} \\
(\mathrm{kpc})\end{array}$ & $\begin{array}{c}D_{T} \\
(\mathrm{kpc})\end{array}$ & $\begin{array}{c}R_{G} \\
(\mathrm{kpc})\end{array}$ & $\begin{array}{c}V_{\mathrm{T}} \\
\left(\mathrm{km} \mathrm{s}^{-1}\right)\end{array}$ & $\mathrm{KDAR}^{\mathrm{a}}$ & $\begin{array}{c}D_{\odot} \\
(\mathrm{kpc})\end{array}$ & $\begin{array}{l}\sigma D_{\odot} \\
(\mathrm{kpc})\end{array}$ & $\begin{array}{r}z \\
\text { (pc) }\end{array}$ \\
\hline G010.621-00.318 & -10.0 & $\ldots$ & 19.8 & 8.4 & 11.6 & 168.7 & $\mathrm{O}$ & 19.8 & 3.1 & -110 \\
\hline G010.630-00.338 & -9.1 & $\ldots$ & 19.5 & 8.4 & 11.2 & 168.7 & $\mathrm{O}$ & 19.5 & 3.0 & -114 \\
\hline S47 & 69.2 & 5.1 & 11.3 & 8.2 & 3.8 & 154.4 & $\mathrm{~N}$ & 5.1 & 0.4 & 16 \\
\hline G019.716-00.261 & 40.1 & 3.3 & 12.7 & 8.0 & 5.5 & 139.9 & $\mathrm{~F}$ & 12.7 & 0.4 & -57 \\
\hline G021.603-00.169a & -4.7 & $\ldots$ & 16.5 & 7.9 & 9.2 & 133.9 & $\mathrm{O}$ & 16.5 & 1.1 & -48 \\
\hline S57 & 31.0 & 2.5 & 13.1 & 7.8 & 6.3 & 129.8 & $\mathrm{~N}$ & 2.5 & 0.5 & 29 \\
\hline G023.295-00.280b & 61.6 & 4.2 & 11.4 & 7.8 & 4.9 & 128.4 & $\mathrm{~F}$ & 11.4 & 0.6 & -55 \\
\hline $\mathrm{G} 025.520+00.215 \mathrm{a}$ & 37.4 & 2.8 & 12.6 & 7.7 & 6.1 & 121.4 & $\mathrm{~F}$ & 12.6 & 0.7 & 47 \\
\hline G025.651-00.031 & 87.5 & 5.3 & 10.0 & 7.7 & 4.4 & 121.0 & $\mathrm{~F}$ & 10.0 & 0.4 & -5 \\
\hline S61 & 37.3 & 2.7 & 12.5 & 7.6 & 6.2 & 118.4 & $\mathrm{~N}$ & 2.7 & 0.4 & 83 \\
\hline
\end{tabular}

Note.

" Kinematic distance ambiguity resolution: "N"-near distance; "F"- - far distance; "O"—outer Galaxy source.

(This table is available in its entirety in machine-readable form.)

source velocity is unknown, as they have at least two possible kinematic distances. We exclude sources in the first and fourth Galactic quadrants for which the absolute value of the tangent point velocity is less than $10 \mathrm{~km} \mathrm{~s}^{-1}$. Finally, we do not provide distances of outer Galaxy regions whose distance uncertainties are $>50 \%$ that of their kinematic distances.

Sources in the inner Galaxy suffer from the well-known kinematic distance ambiguity (KDA): inner Galaxy $\mathrm{H}$ II regions have two possible Heliocentric distances (called "near" and "far") for each measured velocity. The KDA does not exist for the 105 Outer Galaxy H II regions in our sample (including the 34 first-quadrant sources with negative RRL velocities and the four fourth quadrant sources with positive velocities). For all regions, there is no ambiguity in the calculation of Galactocentric distances.

There are 80 inner-Galaxy $\mathrm{H}_{\text {II }}$ regions in our sample for which kinematic distances are possible. We provide a kinematic distance ambiguity resolution (KDAR) for 26 of these (33\%). Four of these are Sharpless regions, which we assume lie at their near distances since they are optically visible (note, however, the extreme distance to S83 discussed later). We analyze the remaining 76 first-quadrant nebulae using the H IE/A method (Anderson \& Bania 2009; Anderson et al. 2012a), employing VGPS $21 \mathrm{~cm}$ data. The HiE/A method uses the fact that $\mathrm{H}_{\mathrm{I}}$ between the observer and the $\mathrm{H}_{\text {II }}$ region is detected in absorption when the brightness temperature of the $\mathrm{H}$ II region plasma at $21 \mathrm{~cm}$ is greater than that of the foreground $\mathrm{HI}_{\mathrm{I}} \mathrm{H}_{\mathrm{I}}$ beyond the $\mathrm{H}$ II region will be seen in emission. This method becomes unreliable if the source velocity is near the tangent point, and therefore we give the six nebulae that have LSR velocities within $10 \mathrm{~km} \mathrm{~s}^{-1}$ of the tangent point the tangent point distance. We are only able to provide a KDAR for 16 of the remaining 70 inner-Galaxy nebulae. To determine distances to the other 54 nebulae we would need more sensitive $\mathrm{H}$ i observations.

We give the kinematic distance parameters for all $131 \mathrm{H}$ II regions with kinematic distances in Table 4 , which lists the source name, the LSR velocity, the near, far, and tangent point distances, the Galactocentric radius, the tangent point velocity, the KDAR ("N"-near distance, "F"-far distance, "T"- tangent point distance, "O"-outer Galaxy), the Heliocentric distance (with uncertainties), and the vertical distance, $z$, from the Galactic mid-plane.
In addition to the kinematic distances described above, there are a number of regions that based on their $(\ell, b, v)$ coordinates are physically near to the Galactic center. We observed four single-velocity regions that are associated with Sgr B2, and four that are associated with Sgr E. These two star forming complexes are physically near to the Galactic center $(\sim 100 \mathrm{pc}$ Liszt 1992; Reid et al. 2009). While we do not provide distance parameters for these sources in Table 5, in later plots we do use a Heliocentric distance of $8.5 \mathrm{kpc}$ and a Galactocentric radius of $0.1 \mathrm{kpc}$ for these eight regions.

\section{DISCUSSION}

\subsection{Sharpless Regions}

The Sharpless regions we detected have velocities similar to those observed in $\mathrm{H} \alpha$ by Fich et al. (1990), although the FWHM line widths are discrepant. We show these comparisons for the $\mathrm{H}_{\text {II }}$ regions with one velocity component in Figure 2. By comparing with the H109 $\alpha$ measurements of Lockman (1989), Fich et al. (1990) also found very good agreement between $\mathrm{H} \alpha$ and RRL velocities, but large discrepancies between the line width measurements. They note a mean $\mathrm{H} \alpha$ and RRL velocity difference of $0.82 \pm 3.46 \mathrm{~km} \mathrm{~s}^{-1}$, whereas we find $0.38 \pm 2.73 \mathrm{~km} \mathrm{~s}^{-1}$ (quoted uncertainties here are the dispersion). The mean absolute velocity difference $\left(\left\langle\left|v_{\mathrm{RRL}}-v_{\mathrm{H} \alpha}\right|\right\rangle\right)$ is $2.02 \pm 1.41 \mathrm{~km} \mathrm{~s}^{-1}$. The $\mathrm{H} \alpha$ line widths are systematically greater than those we measure for RRLs, in agreement with the results of Fich et al. (1990). The average line width ratio $\Delta V_{\mathrm{RRL}} / \Delta V_{\mathrm{H} \alpha}$ is $0.82 \pm 0.17$, whereas Fich et al. (1990) found $0.83 \pm 0.18$. Therefore, the line width discrepancy with $\mathrm{H} \alpha$ is the same, regardless of the observed radio frequency. We hypothesize that the $\mathrm{H} \alpha$ observations are broadened by the relatively low spectral resolution of $\sim 15 \mathrm{~km} \mathrm{~s}^{-1}$. We can spectrally deconvolve the $\mathrm{H} \alpha$ linewidths using $\Delta V_{\text {true }}^{2}=\Delta V_{\mathrm{H} \alpha}^{2}-\Delta V_{\text {Res. }}^{2}$, where the instrumental spectral resolution $V_{\text {Res. }}=15 \mathrm{~km} \mathrm{~s}^{-1}$. Doing so, we find that the average line width ratio $\Delta V_{\mathrm{RRL}} / \Delta V_{\mathrm{H} \alpha}$ is $0.90 \pm 0.24$. The spectral resolution can therefore only explain part of the linewidth difference.

There are 24 Sharpless $\mathrm{H}_{\text {II }}$ regions that have both spectrophotometric distances and kinematics distances derived here. We give the spectrophotometric and kinematic distances for these regions in Table 5 and compare the distances in Figure 3. 
Table 5

Spectrophotometric and Kinematic Distance Comparison

\begin{tabular}{|c|c|c|c|c|c|}
\hline Name & $d_{\text {Spec }}$ & $\begin{array}{c}\sigma d_{\mathrm{S}-} \\
\text { pec }\end{array}$ & $d_{\text {Kin }}$ & $\underset{\mathrm{n}}{\sigma d_{\mathrm{Ki}-}}$ & $\begin{array}{c}\text { Ref- } \\
\text { eren- } \\
\text { ces }\end{array}$ \\
\hline S47 & 2.8 & 0.6 & 3.1 & 0.4 & (1) \\
\hline S57 & 1.7 & 0.5 & 1.5 & 0.5 & (1) \\
\hline S61 & 2.3 & 0.7 & 2.3 & 0.4 & (1) \\
\hline S69 & 3.8 & 1.1 & 3.6 & 0.4 & (1) \\
\hline S92 & 3.9 & 0.5 & 3.7 & 0.1 & (1) \\
\hline S107 & 2.6 & 0.8 & 1.9 & 0.4 & (1) \\
\hline S120 & 6.3 & 1.1 & 10.0 & 1.3 & (1) \\
\hline S121 & 6.3 & 1.1 & 8.5 & 1.2 & (1) \\
\hline S124 & 3.6 & 0.8 & 5.6 & 1.4 & (1) \\
\hline S127 & 9.7 & 2.9 & 12.9 & 1.7 & (1) \\
\hline S128 & 7.8 & 2.3 & 9.2 & 1.5 & (1) \\
\hline S135 & 1.2 & 0.3 & 3.1 & 1.3 & (1) \\
\hline S141 & 8.3 & 0.6 & 7.0 & 1.2 & (2) \\
\hline S148 & 5.3 & 1.1 & 5.8 & 1.3 & (1) \\
\hline S157 & 3.2 & 0.3 & 5.1 & 1.4 & (1) \\
\hline S164 & 2.4 & 0.4 & 4.8 & 1.3 & (1) \\
\hline S168 & 2.5 & 0.5 & 4.4 & 1.3 & (1) \\
\hline S170 & 2.6 & 0.6 & 5.8 & 1.1 & (2) \\
\hline S175 & 2.3 & 0.7 & 5.4 & 1.1 & (2) \\
\hline S193 & 2.4 & 0.3 & 5.2 & 1.3 & (2) \\
\hline S207 & 7.5 & 1.6 & 4.5 & 1.5 & (1) \\
\hline S208 & 7.5 & 1.6 & 3.9 & 1.4 & (1) \\
\hline S219 & 4.0 & 0.7 & 4.9 & 2.1 & (1) \\
\hline S294 & 3.2 & 1.6 & 3.6 & 1.2 & (1) \\
\hline
\end{tabular}

References. (1) Russeil (2003), (2) Russeil et al. (2007).

We take the spectrophotometric distance values and uncertainties from Russeil et al. (2007) if possible, or otherwise from Russeil (2003). While there are more recent spectrophotometric distances for some regions, using distances from these two papers reduces uncertainties caused by different methodologies. The two distances are correlated but there is considerable scatter, especially for sources with small spectrophotometric distances. The mean difference $\left(\left\langle d_{\text {Kin }}-d_{\text {Spec }}\right\rangle\right)$ between kinematic and spectrophotometric distances is $1.0 \pm 2.0 \mathrm{kpc}$. As a percentage of the spectrophotometric distances, the differences are on average $47 \pm 90 \%$ discrepant. The average absolute difference $\left(\left\langle\left|d_{\text {kin }}-d_{\text {spec }}\right|\right\rangle\right)$ in kinematic and spectrophotometric distances is $1.8 \pm 1.3 \mathrm{kpc}$. The agreement is improved if the Reid et al. (2014) rotation curve is used.

The largest discrepancies between distances are for $\mathrm{H}_{\mathrm{II}}$ regions with small spectrophotometric distances in the zone $136^{\circ}>\ell>105^{\circ}$. For example, S135, S164, S170, S175, and S193 all have spectrophotometric distances $<2.5 \mathrm{kpc}$, but kinematic distances from the Brand \& Blitz (1993) rotation curve $\geqslant 100 \%$ discrepant. The $(\ell, b, v)$ locii of these regions is suggests that they lie in the Perseus spiral arm where perhaps non-circular motions are leading to erroneously large kinematic distances (Choi et al. 2014). This explanation assumes that the spectrophotometric distances are more accurate than the kinematic distances, which has not been proven to be the case. We do note that for S170 the maser parallax distance of $3.34 \mathrm{kpc}$ (Choi et al. 2014) is in better agreement with the spectrophotometric distance of $2.6 \mathrm{kpc}$ compared with our kinematic distance of $5.8 \mathrm{kpc}$. Choi et al. (2014) list kinematic distances for this source of 3.0 and $4.0 \mathrm{kpc}$ for different rotation curve models based on their maser parallax distances, which
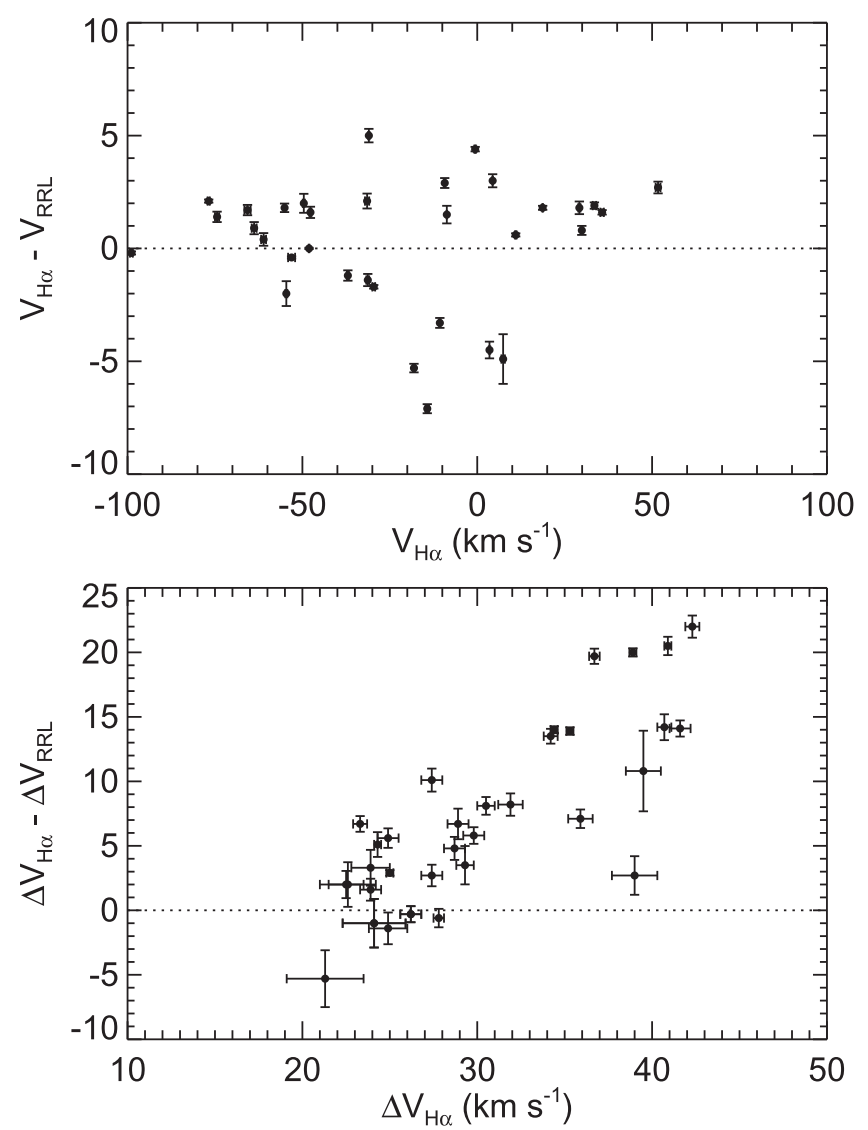

Figure 2. Comparison of $\mathrm{H} \alpha$ (from Fich et al. 1990) and RRL velocities (top) and FWHM line widths (bottom) for observed Sharpless $\mathrm{H}$ il regions. The velocity differences are small compared to the $\sim 15 \mathrm{~km} \mathrm{~s}^{-1} \mathrm{H} \alpha$ velocity resolution and there is no systematic offset. The line widths, however, are significantly broader for the $\mathrm{H} \alpha$ observations, in agreement with the finding of Fich et al. (1990).

implies that the Brand \& Blitz (1993) curve dramatically overestimates kinematic distances in this direction.

\subsection{Galactic Distribution}

Here, we have added significant numbers of $\mathrm{H}$ II regions to the Galactic census, especially in the sky zones not covered by the original HRDS. In Figure 4 we show the distribution of regions detected here and of all $\mathrm{H}$ II regions known previously. The sample of previously known regions all have ionized gas velocities (RRL or $\mathrm{H} \alpha$ ), as compiled in the WISE catalog (A14). The sample here adds considerably to the $\mathrm{H}$ II region census in the portion of the first Galactic quadrant not covered by the original HRDS, $90^{\circ}>\ell>65^{\circ}$, with 42 new detections versus 52 regions known previously. We also add 105 outer Galaxy regions (mostly in the second quadrant) versus 160 known previously (Table 4).

As expected, the regions in the current sample are more distant on average than the population known previously. To illustrate this point, in Figure 5 we show the distributions of Heliocentric and Galactocentric distances for the current census of Galactic $\mathrm{H}_{\mathrm{II}}$ regions. The average Heliocentric and Galactocentric distances for the new detections are 9.8 and $11.0 \mathrm{kpc}$, respectively, while they are 7.4 and $6.8 \mathrm{kpc}$ for the previously known sample compiled by A14. Here we provide kinematic distances for 60 newly found first-quadrant regions. The average Heliocentric distance to these 60 regions is 


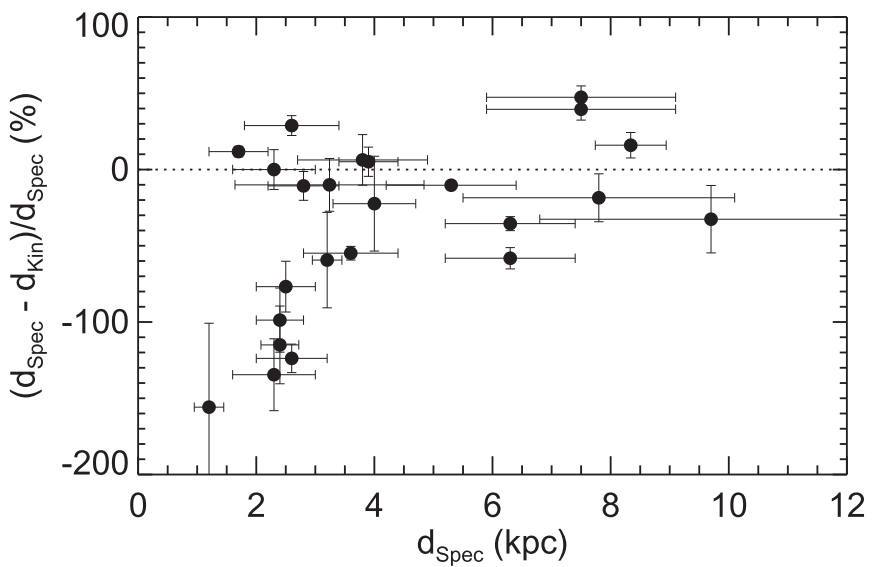

Figure 3. Comparison of the percentage difference between spectrophotometric distances and kinematic distances derived here. The differences are large on average, but are generally less than $100 \%$ discrepant. The largest discrepancies are for sources with small spectrophotometric distances.

$15.2 \mathrm{kpc}$, whereas it is $10.1 \mathrm{kpc}$ for the first-quadrant regions in the original HRDS (Anderson et al. 2012a) and $8.4 \mathrm{kpc}$ for the first-quadrant regions known prior to the HRDS (Anderson et al. 2009). The average Galactocentric radius for the 60 firstquadrant sources is $9.4 \mathrm{kpc}$. Of the 60 regions, 34 have negative RRL velocities and are therefore in the outer Galaxy. In the second quadrant, the average distance is $7.2 \mathrm{kpc}$, whereas it was $5.7 \mathrm{kpc}$ for regions known previously. We attribute these differences to the fact that we explicitly searched for distant regions following the warp in the first quadrant, and observed fainter sources than were known previously in the second quadrant.

\subsection{Outer Scutum Centaurus Arm}

We detected the RRL emission from $47 \mathrm{H}$ II regions in the $(\ell, b)$ zone of the OSC searched here: $65^{\circ}>\ell>25^{\circ} ; b \geqslant 1^{\circ}$. Of these $47 \mathrm{H}$ II regions, five have LSR velocities within $15 \mathrm{~km} \mathrm{~s}^{-1}$ of the OSC $(\ell, v)$ locus defined by in Dame \& Thaddeus (2011): $v=-1.6 \times \ell$. Included in this tally is S83, whose RRL velocity was already known to be consistent with the OSC (e.g., Balser et al. 2011, where it is called "G55.11 +2.4 "). We also detect the RRL emission from an additional region not in the $\operatorname{OSC}(\ell, b)$ zone whose longitude and velocity are nevertheless consistent with membership in the OSC, G039.183-01.422 (see below), bringing the total number of $\mathrm{H}$ II regions identified here that appear to be in the OSC to 6: G033.008+01.151, G039.183-01.422, G041.755+01.451, G041.804+01.503, G054.094+01.749, and S83. The regions G041.755+01.451 and G041.804+01.503 have small angular separations and have RRL velocities within $\sim 2 \mathrm{~km} \mathrm{~s}^{-1}$ of each other. They are therefore presumably part of the same complex.

We give the parameters of the OSC regions in Table 5, where we list Galactocentric and Heliocentric distances according to the Brand \& Blitz (1993; with $\Theta_{0}=220 \mathrm{~km} \mathrm{~s}^{-1}$ and $R_{0}=8.5 \mathrm{kpc}$ ) and Reid et al. (2014) rotation curves (with $\Theta_{0}=240 \mathrm{~km} \mathrm{~s}^{-1}$ and $R_{0}=8.34 \mathrm{kpc}$ ). The Reid et al. (2014) curve gives distances $\sim 10 \%$ smaller for the OSC sources, on average $\sim 2 \mathrm{kpc}$ for distances of $\sim 20 \mathrm{kpc}$, due to the larger rotational speeds of the model.

The source G039.183-01.422 has an LSR velocity consistent with being in the OSC, and yet according to its kinematic distance it lies nearly 500 pc below the Galactic plane. At $\ell=39^{\circ}$, the $\mathrm{H}_{\mathrm{I}}$ in the OSC is centered near $b=3^{\circ}$ (c.f. Dame \& Thaddeus 2011) or $\sim 600$ pc above the Galactic mid-plane at a Heliocentric distance of $\sim 20 \mathrm{kpc}$. There is only a small amount of OSC $\mathrm{H}_{\mathrm{I}}$ emission seen at the $(\ell, b)$ position of G039.183-01.422 (see Dame \& Thaddeus 2011). The implied separation of G039.183-01.422 from the OSC arm centroid therefore corresponds to a vertical offset of $>1 \mathrm{kpc}$. It is unlikely that the high-mass stars capable of producing $\mathrm{H}$ II regions would have strayed this far from their birthplaces. At the nominal lower velocity for a star to be called a "runaway," $30 \mathrm{~km} \mathrm{~s}^{-1}$, it would take over $30 \mathrm{Myr}$ to travel $1 \mathrm{kpc}$. This neglects motion in the plane of the sky. Of course, if it is indeed a runaway star, its velocity cannot be used for kinematic distances. It is possible that G039.183-01.422 is a planetary nebula, but we regard this as unlikely due to the detection of an $\mathrm{H}_{2} \mathrm{O}$ maser at $-53 \mathrm{~km} \mathrm{~s}^{-1}$ (Sunada et al. 2007) as well as $\mathrm{NH}_{3}$ (W. Armentrout, 2015, in preparation).

\subsection{Distant Outer Galaxy Sources Toward $\ell=150^{\circ}$}

Three second-quadrant sources have Galactocentric radii from the Brand \& Blitz (1993) rotation curve that are greater than 19kpc: G149.746-00.199, G151.561-00.425, and G151.626-00.456. Based on their WISE emission, the latter two sources appear to be in the same complex as S209, which has a RRL velocity of $-51.2 \mathrm{~km} \mathrm{~s}^{-1}$ (Balser et al. 2011). Properties of these nebulae are included in Table 6. The Reid et al. (2014) curve gives Galactocentric distances lower by $3-5 \mathrm{kpc}(\sim 20 \%$; Table 6$)$. Regardless of their exact distances, these sources have the largest Galactocentric radii of any $\mathrm{H}$ II regions discovered and are likely located in the extreme outer Galaxy (EOG Kobayashi et al. 2008; Yasui et al. 2008). With low metallicity and low densities, the EOG is a laboratory for how stars may have formed in the early evolutionary stages of our Milky Way.

The three distant sources may be part of the OSC extension into the second Galactic quadrant that was identified by Sun et al. (2015), whose analysis shows $\mathrm{H}_{\mathrm{I}}$ emission at $(\ell, v)=\left(\sim 150^{\circ}, \sim-70 \mathrm{~km} \mathrm{~s}^{-1}\right)$. While the $\mathrm{H}$ in region velocities are in rough agreement with this $(\ell, v)$ locus, the brightest $\mathrm{H}_{\mathrm{I}}$ emission near $\ell \simeq 150^{\circ}$ is at $b \simeq-3^{\circ}$ whereas our sources are much closer to $b=0^{\circ}$. It is therefore not clear at present if these three H II regions (and S209) lie in the OSC. These distant $\mathrm{H}_{\mathrm{II}}$ regions are not spatially coincident with any of the molecular clouds detected by Digel et al. (1994), which have Galactic longitudes of $130^{\circ}-150^{\circ}$ and Galactocentric radii $\gtrsim 17 \mathrm{kpc}$.

\subsection{Nuclear Disk (Sgr E)}

We detected RRL emission from five $\mathrm{H}_{\text {II }}$ regions near $\ell=359^{\circ}$ that have negative velocity components $\lesssim-200 \mathrm{~km} \mathrm{~s}^{-1}: \quad$ G358.600-00.057, G358.643-00.034, G358.684-00.116, G358.802-00.011, and G358.946 +00.004 . These nebulae are part of Sgr E, a well-known complex of H II regions near the Galactic center (Liszt 1992) that contains at least 30 separate sources identified from their spectral indices (Gray et al. 1993). Few of these 30 regions have ionized gas spectroscopic measurements. Sgr E is thought to be at the outer boundary of the nuclear disk, which implies a distance from the Galactic center of $\sim 200 \mathrm{pc}$. It has no counterpart at positive longitudes. Sgr E was previously observed in RRL emission by Cram et al. (1996), and they 


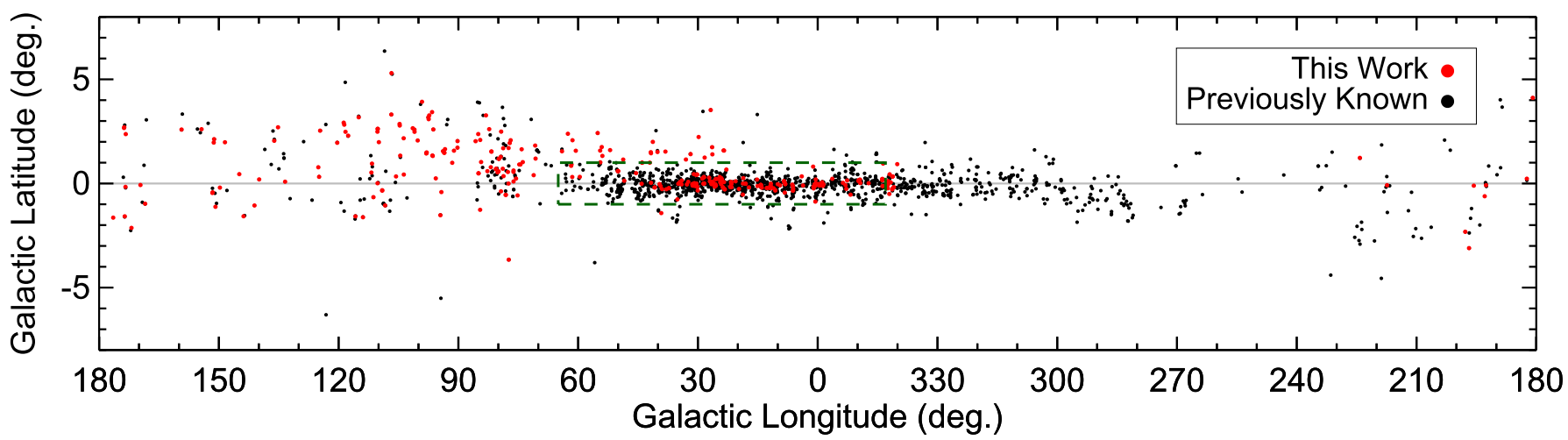

Figure 4. Galactic distribution of the detected $\mathrm{H}$ II regions (red) and those known previously (black). The previously known sample is from A14 and includes regions from the original HRDS, whose survey zone, $65^{\circ}>\ell>-17^{\circ} ;|b| \leqslant 0^{\circ}$, is indicated by the dashed box. Outside the range of the original HRDS the new detections are especially numerous compared to the sample of previously known $\mathrm{H}$ II regions, with 170 new detections over the survey area vs. 214 regions known previously. The gray horizontal line indicates $b=0^{\circ}$. The new detections have a broader latitude distribution in the first quadrant than those previously known.
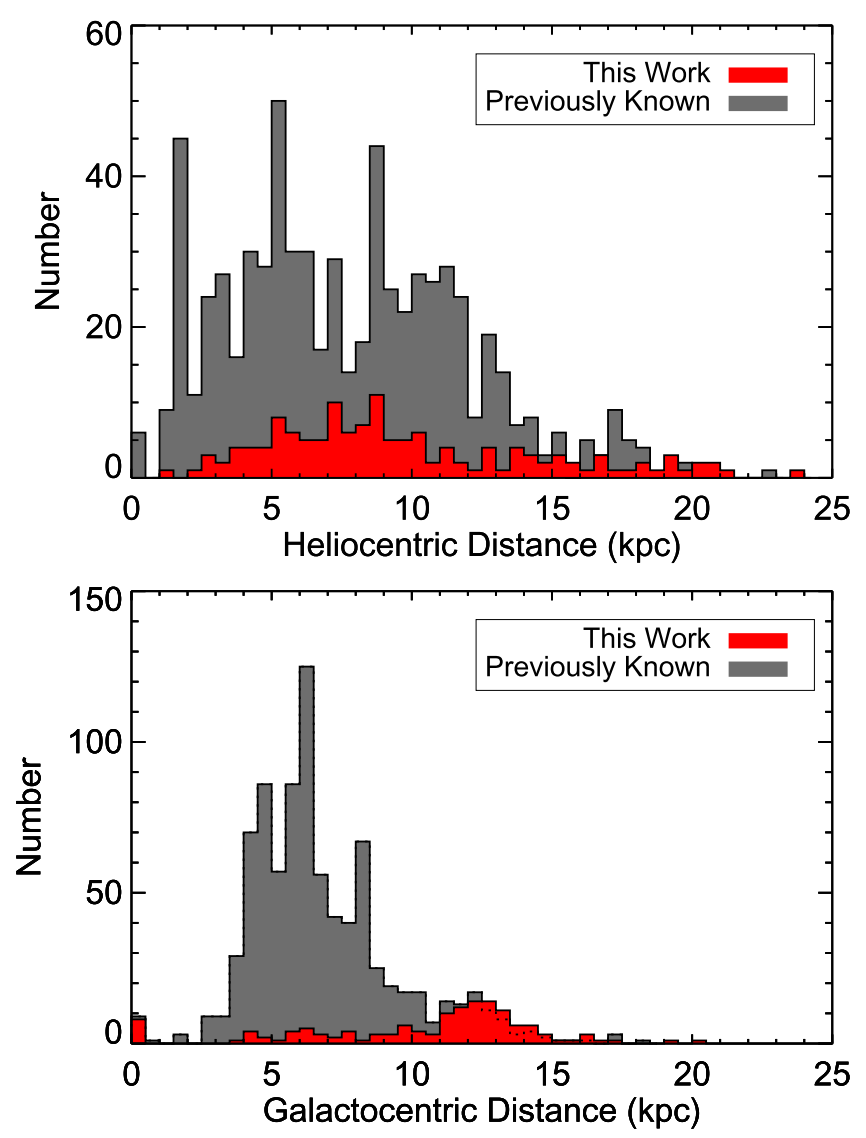

Figure 5. Heliocentric (top) and Galactocentric (bottom) $\mathrm{H}$ II region distance distributions for the new detections (red) and those previously known (gray). The previously known sample contains all $\mathrm{H}$ II regions with a single measured ionized gas velocity component (H $\alpha$ or RRLs) compiled in the WISE catalog of A14, incuding regions from the original HRDS. Here we show all nebulae located in the zone $\left(225^{\circ}>\ell>-20^{\circ}\right)$. Neither sample shown here contains sources with multiple velocity components. The newly detected $\mathrm{H}$ II regions are more distant on average than those known previously, in terms of both Heliocentric and also Galactocentric distances. The average Heliocentric and Galactocentric distances for the new detections are 9.8 and $11.0 \mathrm{kpc}$, respectively, while they are 7.4 and $6.8 \mathrm{kpc}$ for the previously known sample.

detected six $\mathrm{H}$ II regions, including four of the regions detected here. The region G358.946+00.004, which was not detected by Cram et al. (1996), has multiple RRL velocities and therefore its membership in Sgr E is less secure.
On the basis of their Galactic location and LSR velocities near $-200 \mathrm{~km} \mathrm{~s}^{-1}$, Sgr E now has $16 \mathrm{H}$ II regions with measured RRL emission, including nine regions from the original HRDS (one of which, G358.720+0.011, was also observed by Cram et al. 1996). There are two additional $\mathrm{H}_{\text {II }}$ regions near $(\ell, b)=\left(359^{\circ}, 0^{\circ}\right)$ with velocities near $0 \mathrm{~km} \mathrm{~s}^{-1}$ whose membership in Sgr E is unlikely: G358.881+00.057; $-7.0 \mathrm{~km} \mathrm{~s}^{-1}$ (detected here) and G358.633+00.062; $14.0 \mathrm{~km} \mathrm{~s}^{-1}$ (detected by A11). We show the infrared emission from the Sgr E region in Figure 6, which has $24 \mu \mathrm{m}$ Spitzer MIPSGAL data in red, $8.0 \mu \mathrm{m}$ Spitzer GLIMPSE data in green, and $3.6 \mu \mathrm{m}$ Spitzer GLIMPSE data in blue.

The morphology of the Sgr E H II nebulae is unusual in that their $8.0 \mu \mathrm{m}$ emission is weak compared to their $24 \mu \mathrm{m}$ emission. While there is faint $8.0 \mu \mathrm{m}$ emission surrounding each source, the strongest $8.0 \mu \mathrm{m}$ emission is found toward the center of the $\mathrm{H}$ II regions. This unusual infrared morphology is not seen outside of the Galactic center. Furthermore, for the two regions whose velocities are not consistent with Sgr E, the $8.0 \mu \mathrm{m}$ emission is strong, and found surrounding the regions.

The integrated 8.0 to $24 \mu \mathrm{m}$ ratios are on average $\sim 3$ times less than the Galactic average for $\mathrm{H}$ II regions from Anderson et al. (2012b). Why the $8.0 \mu \mathrm{m}$ emission in the Sgr E H II regions is so weak is unknown. The $8.0 \mu \mathrm{m}$ emission may be attenuated by intervening material, the emission process that produces the $8.0 \mu \mathrm{m}$ emission may be absent for Sgr E, or the photodissociation regions surrounding the Sgr E regions may not be present.

\section{SUMMARY}

We report the GBT detection of $302 \mathrm{H}$ II regions in RRL and radio continuum emission. These regions were identified as $\mathrm{H}$ II region candidates from their mid-infrared and radio continuum emission in the WISE Catalog of Galactic H II Regions (Anderson et al. 2014), and have estimated peak flux densities $>30 \mathrm{mJy}$ in the X-band. The regions are distributed throughout the entire sky visible from the GBT $\left(220^{\circ}>\ell>-20^{\circ}\right.$ at $b=0^{\circ}$ ). The largest number of detections is in areas of the sky not studied in our previous HRDS, namely $|b|>1^{\circ}, \ell<-17^{\circ}$, and $\ell>65^{\circ}$. In these zones the present survey has roughly doubled the number of known $\mathrm{H}$ II regions, bringing the total number of $\mathrm{H}$ II regions detected in our RRL surveys to nearly 800 . Here, we provide kinematic distances for 131 regions. 
Table 6

OSC H II Regions?

\begin{tabular}{|c|c|c|c|c|c|c|c|c|c|}
\hline \multirow[b]{2}{*}{ Name } & \multirow[b]{2}{*}{$\begin{array}{c}\ell \\
\left({ }^{\circ}\right)\end{array}$} & \multirow[b]{2}{*}{$\begin{array}{c}b \\
\left({ }^{\circ}\right)\end{array}$} & \multirow[b]{2}{*}{$\begin{array}{c}V_{\mathrm{LSR}} \\
\left(\mathrm{km} \mathrm{s}^{-1}\right)\end{array}$} & \multicolumn{3}{|c|}{ Brand } & \multicolumn{3}{|c|}{ Reid } \\
\hline & & & & $\begin{array}{c}R_{G} \\
(\mathrm{kpc})\end{array}$ & $\begin{array}{c}d_{\odot} \\
(\mathrm{kpc})\end{array}$ & $\begin{array}{r}z \\
(\mathrm{pc})\end{array}$ & $\begin{array}{c}R_{G} \\
(\mathrm{kpc})\end{array}$ & $\begin{array}{c}d_{\odot} \\
(\mathrm{kpc})\end{array}$ & $\begin{array}{r}z \\
\text { (pc) }\end{array}$ \\
\hline G033.008+01.151 & 33.008 & 1.151 & -57.6 & 17.0 & 23.5 & 472 & 14.8 & 21.1 & 424 \\
\hline G039.183-01.422 & 39.183 & -1.422 & -54.9 & 14.5 & 20.1 & -499 & 13.0 & 18.4 & -456 \\
\hline G041.755+01.451 & 41.755 & 1.451 & -54.8 & 14.0 & 19.2 & 485 & 12.6 & 17.6 & 445 \\
\hline G041.804+01.503 & 41.804 & 1.503 & -52.6 & 13.7 & 18.8 & 493 & 12.4 & 17.3 & 453 \\
\hline G054.094+01.749 & 54.094 & 1.749 & -85.3 & 17.0 & 20.5 & 626 & 14.8 & 18.0 & 550 \\
\hline S83 & 55.114 & 2.422 & -76.1 & 15.3 & 18.4 & 778 & 13.5 & 16.5 & 695 \\
\hline G149.746-00.199 & 149.746 & -0.199 & -71.4 & 25.3 & 17.5 & -60 & 20.2 & 12.5 & -43 \\
\hline G151.561-00.425 & 151.561 & -0.425 & -56.4 & 19.3 & 11.4 & -84 & 16.4 & 8.5 & -63 \\
\hline G151.626-00.456 & 151.626 & -0.456 & -58.2 & 20.1 & 12.2 & -97 & 16.9 & 9.1 & -72 \\
\hline
\end{tabular}

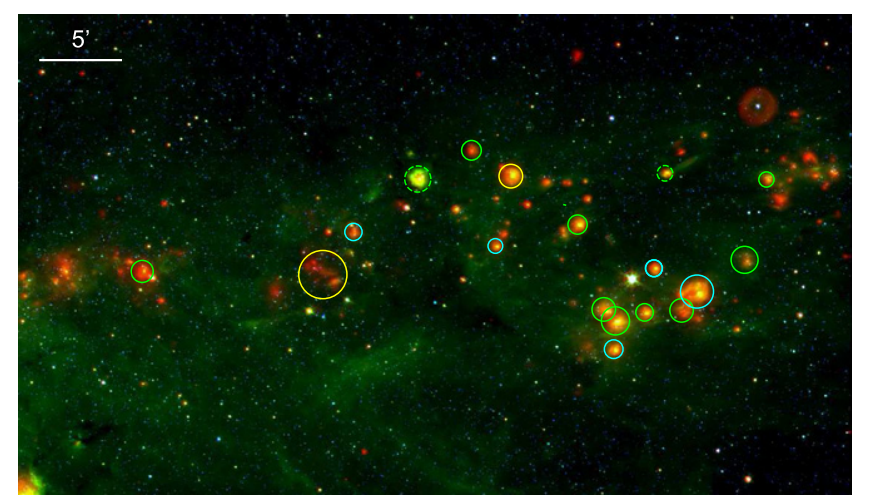

Figure 6. Spitzer image of nuclear disk $\mathrm{H}$ II regions in Sgr E. The field is $50^{\prime} \times 30^{\prime}$, centered at $(\ell, b)=\left(359^{\circ} 863,-0.020\right)$. The $24 \mu \mathrm{m}$ MIPSGAL data are red, the $8.0 \mu \mathrm{m}$ GLIMPSE data are in green, and the $3.6 \mu \mathrm{m}$ GLIMPSE data are in blue. Circles approximate the $\mathrm{H}$ II region infrared sizes and are color-coded such that cyan circles enclose $\mathrm{H}$ II regions from this work, green are from the original HRDS (A11), and yellow are from Lockman et al. (1996). The two dashed circles surround regions that have velocities inconsistent with that of the nuclear disk. Other sources evident in the field lack ionized gas spectroscopic velocities. The nuclear disk sources have ratios of 8.0 to $24 \mu \mathrm{m}$ fluxes about three times less than those of $\mathrm{H}$ II regions found in the rest of the Galaxy.

Many of the new detections are extremely distant. We detect five $\mathrm{H}$ II regions that have $(\ell, b, v)$ coordinates consistent with the OSC, and one additional region with a velocity consistent with the OSC but at a negative Galactic latitude (G039.183 $\left.-01.422 ;-54.9 \mathrm{~km} \mathrm{~s}^{-1}\right)$. The OSC is the most distant molecular spiral arm of the Milky Way and these $\mathrm{H}$ II regions are the most distant ever discovered. The OSC regions' Heliocentric distances are $>22 \mathrm{kpc}$, and their Galactocentric distances are $>16 \mathrm{kpc}$. The OSC regions were missed in previous surveys of the Galactic plane because the OSC is found at Galactic latitudes $>1^{\circ}$ in the first Galactic quadrant. We detect an additional three $\mathrm{H}$ II regions near $\ell \simeq 150^{\circ}$ whose LSR velocities imply Galactocentric distances $>19 \mathrm{kpc}$. These nebulae may be part of an extension of the OSC, and they may represent the limit to Galactic massive star formation.

The National Radio Astronomy Observatory is a facility of the National Science Foundation operated under cooperative agreement by Associated Universities, Inc. This work supported by NASA ADAP grant NNX12AI59G and NSF grant AST1516021. We thank the staff at the Green Bank Telescope for their hospitality and friendship during the observations and data reduction. We thank West Virginia University for its financial support of GBT operations, which enabled some of the observations for this project.

Facility: Green Bank Telescope.

\section{APPENDIX \\ WEB SITES}

We have updated the GBT HRDS website with results from this work. ${ }^{8}$ This site contains images such as those in Figure 1 for all detected sources, as well as the same for the sources from A11 and Bania et al. (2012). We have also updated the WISE Catalog of Galactic $\mathrm{H}$ II Regions web site ${ }^{9}$ with results from these observations.

\section{REFERENCES}

Anderson, L. D., \& Bania, T. M. 2009, ApJ, 690, 706

Anderson, L. D., Bania, T. M., Balser, D. S., et al. 2014, ApJS, 212, 1

Anderson, L. D., Bania, T. M., Balser, D. S., \& Rood, R. T. 2011, ApJS, 194, 32

Anderson, L. D., Bania, T. M., Balser, D. S., \& Rood, R. T. 2012a, ApJ, 754,62

Anderson, L. D., Bania, T. M., Jackson, J. M., et al. 2009, ApJS, 181, 255

Anderson, L. D., Hough, L. A., Wenger, T. V., Bania, T. M., \& Balser, D. S. 2015, ApJ, 810, 42

Anderson, L. D., Zavagno, A., Barlow, M. J., García-Lario, P., \& Noriega-Crespo, A. 2012b, A\&A, 537, A1

Balser, D. S. 2006, AJ, 132, 2326

Balser, D. S., Rood, R. T., Bania, T. M., \& Anderson, L. D. 2011, ApJ, 738, 27

Bania, T. M., Anderson, L. D., \& Balser, D. S. 2012, ApJ, 759, 96

Bania, T. M., Anderson, L. D., Balser, D. S., \& Rood, R. T. 2010, ApJL, 718, L106

Bania, T., Wenger, T., Balser, D., \& Anderson, L. 2014, tmbidl: TMBIDL v7.1, Zenodo (10.5281/zenodo.32790)

Bock, D., Large, M. I., \& Sadler, E. M. 1999, AJ, 117, 1578

Brand, J., \& Blitz, L. 1993, A\&A, 275, 67

Carey, S. J., Noriega-Crespo, A., Mizuno, D. R., et al. 2009, PASP, 121, 76

Choi, Y. K., Hachisuka, K., Reid, M. J., et al. 2014, ApJ, 790, 99

Condon, J. J., Cotton, W. D., Greisen, E. W., et al. 1998, AJ, 115, 1693

Cram, L. E., Claussen, M. J., Beasley, A. J., Gray, A. D., \& Goss, W. M. 1996, MNRAS, 280, 1110

Dame, T. M., \& Thaddeus, P. 2011, ApJL, 734, L24

Digel, S., de Geus, E., \& Thaddeus, P. 1994, ApJ, 422, 92

Fich, M., Dahl, G. P., \& Treffers, R. R. 1990, AJ, 99, 622

Ghigo, F., Maddalena, R., Balser, D., \& Langston, G. 2001, GBT Commissioning Memo 10

Gordon, M. A. 1976, in Methods of Experimental Physics, Vol. 12, ed. M. L. Meeks (New York: Academic)

Gray, A. D., Whiteoak, J. B. Z., Cram, L. E., \& Goss, W. M. 1993, MNRAS, 264,678

\footnotetext{
8 http://go.nrao.edu/hrds

9 http://astro.phys.wvu.edu/wise
} 
Kobayashi, N., Yasui, C., Tokunaga, A. T., \& Saito, M. 2008, ApJ, 683, 178

Kuchar, T. A., \& Clark, F. O. 1997, ApJ, 488, 224

Liszt, H. S. 1992, ApJS, 82, 495

Lockman, F. J. 1989, ApJS, 71, 469

Lockman, F. J., Pisano, D. J., \& Howard, G. J. 1996, ApJ, 472, 173

McClure-Griffiths, N. M., Dickey, J. M., Gaensler, B. M., et al. 2005, ApJS, 158,178

Peng, B., Kraus, A., Krichbaum, T. P., \& Witzel, A. 2000, A\&AS, 145, 1

Quireza, C., Rood, R. T., Balser, D. S., \& Bania, T. M. 2006a, ApJS, 165,338

Quireza, C., Rood, R. T., Bania, T. M., Balser, D. S., \& Maciel, W. J. 2006b, ApJ, 653, 1226

Reid, M. J., Menten, K. M., Brunthaler, A., et al. 2014, ApJ, 783, 130
Reid, M. J., Menten, K. M., Zheng, X. W., Brunthaler, A., \& Xu, Y. 2009, ApJ, 705,1548

Russeil, D. 2003, A\&A, 397, 133

Russeil, D., Adami, C., \& Georgelin, Y. M. 2007, A\&A, 470, 161

Sharpless, S. 1959, ApJS, 4, 257

Stil, J. M., Taylor, A. R., Dickey, J. M., et al. 2006, AJ, 132, 1158

Sun, Y., Xu, Y., Yang, J., et al. 2015, ApJL, 798, L27

Sunada, K., Nakazato, T., Ikeda, N., et al. 2007, PASJ, 59, 1185

Taylor, A. R., Gibson, S. J., Peracaula, M., et al. 2003, AJ, 125, 3145

Taylor, J. H., \& Cordes, J. M. 1993, ApJ, 411, 674

Wenger, T. V., Bania, T. M., Balser, D. S., \& Anderson, L. D. 2013, ApJ, 764,34

Yasui, C., Kobayashi, N., Tokunaga, A. T., Terada, H., \& Saito, M. 2008, ApJ, 675,443 